Dossier Interdisciplinarité

\title{
L'institutionnalisation de l'interdisciplinarité au sein de l'Université brésilienne : le cas des sciences de l'environnement
}

\author{
Marcel Bursztyn \\ Économiste social, Université de Brasilia, CDS-SAS, quadra 5, bloco H, $2^{\circ}$ andar CEP 70.070-914, Brasília, Brésil
}

\author{
Mots-clés : \\ environnement ; \\ développement \\ durable ; \\ interdisciplinarité; \\ université ; \\ Brésil
}

\section{Keywords: environment; sustainable development; interdisciplinarity; university; Brazil}

\begin{abstract}
Résumé - Depuis quelques décennies, la production de connaissances dans le milieu académique est marquée par une notable tendance à la spécialisation. La question de l'environnement et du développement durable, qui est très présente, provoque un débat sur la nécessité d'établir des espaces interdisciplinaires au sein des universités. Au Brésil, la récente insertion des formations interdisciplinaires au niveau du master et du doctorat a lieu dans un contexte institutionnel difficile, qui est l'expression de la crise générale de l'Université dans ce pays. Le texte présente des expériences novatrices de mise en place de formations sur le développement durable, met en évidence les obstacles et rend compte des stratégies pour leur généralisation dans les universités, leur validation et leur accréditation. En conclusion, l'idée d'une Université-réseau apparaît comme une formule possible.
\end{abstract}

\begin{abstract}
Institutionalization of interdisciplinarity in the Brazilian university: the case of environmental sciences. Production of academic knowledge in the past two centuries has been marked by a clear trend towards specialization. The considerable importance currently gained by environmental issues, as seen through the lense of sustainable development, has promoted significant changes in this scenario and highlighted the need for interdisciplinary approaches (de-specialization). The recent creation of interdisciplinary master's and doctoral training programs in Brazil faces a range of obstacles related to the general crisis prevailing in University. Using examples taken from Brazilian graduate programs, our paper outlines and analyzes critically the programs focused on environmental issues nationwide and shows the significant limitations and challenges incurred in building this new field of knowledge. Despite a growing trend towards "environmentalization" of some disciplines, the issue at stake is to interdisciplinarize the focus on sustainable development. The consolidated programs are presented according to the specific institutional arrangements adopted by them. They clearly reflect a need to act creatively in order to break through the impenetrable structure of disciplinary departments. To conlcude, the idea of building a network structure within University is argued as a possible alternative, a solution that does not deny the role of traditional departments, but opens spaces for their interaction with interdisciplinary units and programs.
\end{abstract}

« Le concept de science sur lequel nous vivons n'est ni absolu, ni éternel; la science évolue [...] une révolution est sur le point de regrouper les disciplines... »

E. Morin (2004)

Auteur correspondant : marcel@unb.br

M. Bursztyn est détenteur d'un master en planification urbaine et régionale et d'un doctorat en développement économique et social. Il est actuellement professeur au Centre de développement durable et au département de Sociologie de l'université de Brasília. Il a été fonctionnaire du CNPq, président de la CAPES et du Fonds d'appui à la recherche du Distrito Federal (voir en note 1 l'explication des sigles CNPq et CAPES).
La question de l'interdisciplinarité au sein de l’Université brésilienne est ancienne. Mais elle est devenue plus pressante à la fin du XXe siècle. On l'abordera ici à propos des enseignements de master et de doctorat ayant trait à l'environnement et au développement durable. Le cas brésilien est, jusqu'à un certain degré, l'expression d'un phénomène vécu également par d'autres pays.

Plusieurs expériences, qui se distinguent par leurs stratégies d'implantation créatives, seront évoquées. Elles mettent en évidence un point commun : une légitimation rendue difficile par des pratiques enracinées 
de partage du pouvoir entre des corporations qui interviennent selon des règles égocentriques. Il en résulte, pour les expériences en cours, des contraintes plus ou moins fortes, dont on peut dégager un certain nombre de caractéristiques générales.

\section{Expériences interdisciplinaires au Brésil : remonter aux années soixante-dix}

Les expériences d'institutionnalisation des structures interdisciplinaires au sein des universités brésiliennes se sont peu à peu multipliées à partir des années quatrevingt-dix. Toutefois, l'analyse ne peut se restreindre à cette période. Plusieurs cas remarquables et couronnés de succès ont été enregistrés, principalement à partir des années soixante-dix.

Un exemple particulièrement significatif est la création, avec le soutien du gouvernement fédéral, d'enseignements de master en "planification urbaine et régionale ». L'Université brésilienne vivait alors une «réforme » d'inspiration internationale et post-soixantehuitarde : un système de départements et de crédits, avec autonomisation des élèves par rapport aux cours dispensés, et une déconcentration des campus.

L'interdisciplinarité - encore qu'à l'époque, celle-ci n'ait suscité ni débats ni même oppositions - est une caractéristique des programmes d'études de $2^{\mathrm{e}}$ ou de $3^{\mathrm{e}}$ cycle en planification urbaine et régionale. Le fait que cet enseignement fasse son apparition dans un contexte de changements institutionnels placé sous le signe de la répression (en pleine dictature militaire) et de l'absence de débats peut avoir été un facteur favorable. Mais il faut également tenir compte du fait que le profil institutionnel adopté $\mathrm{n}$ 'impliquait aucune perspective d'interaction entre différents départements. Ces programmes, de fait pluridisciplinaires en raison de la variété de formation de leurs enseignants, se mettaient en place comme s'il s'agissait d'une discipline en soi : en somme, une «discipline interdisciplinaire ».

Par certains aspects, le contexte universitaire favorise cette institutionnalisation des expériences interdisciplinaires dans les années soixante-dix. Tout d'abord, il convient de considérer qu'à cette époque, l'Université n'était pas aussi fragmentée qu'actuellement. Le nombre de disciplines qui formait l'univers des enseignements était bien plus réduit. Dans les sciences sociales, par exemple, la séparation entre l'anthropologie et la sociologie n'avait pas encore eu lieu. Les cours d'ingénierie ne comportaient pas autant de spécialités. Dans de nombreux cas, différents enseignements possédaient un tronc commun, ou partaient d'un même cycle de base.

Ensuite, l'exercice du pouvoir, peut-être parce qu'il était récent (au Brésil, les enseignements des $2^{\mathrm{e}}$ et $3^{\mathrm{e}}$ cycles s'organisaient régulièrement et selon des critères d'accréditation et d'évaluation au long des années soixante-dix), ne brimait pas ces nouvelles initiatives, bien au contraire. De surcroît, l'Université faisait figure de «victime» du régime militaire, ce qui stimulait une solidarité systématique entre ses protagonistes.

Durant le régime militaire, le fonctionnement des universités (publiques) était plutôt assuré sur le plan matériel. Les enseignants revendiquaient périodiquement davantage de ressources, mais le cadre budgétaire, fixé alors en fonction du nombre d'élèves et des activités de recherche, était moins pénalisant que celui qui se présenterait au cours des décennies suivantes. Il n'y avait donc pas de concurrence pour l'obtention de moyens financiers. Ne suscitant pas d'opposition particulière, les nouvelles initiatives étaient même encouragées, car elles entraînaient la diversification de l'offre de formation et l'augmentation du nombre des places, ce que souhaitaient des universités encore jeunes. Les domaines disciplinaires consolidés étaient davantage préoccupés par leur propre structuration face à une réforme en cours que par ces nouvelles initiatives.

$\mathrm{Au}$ fil du temps, les cours de planification urbaine et régionale ont été suffisamment solides pour survivre à l'arrêt des soutiens gouvernementaux. La création de comités d'évaluation spécifiques, au sein de la CAPES et $\mathrm{du} \mathrm{CNPq}{ }^{1}$ les confirme comme domaine de connaissance spécifique ${ }^{2}$.

L'apparition de programmes ayant trait à « l'environnement » et au « développement durable » et, plus récemment, à la «gérontologie », reproduit partiellement l'expérience de la "planification urbaine et régionale », mais dans un environnement institutionnel assez différent de celui des années soixante-dix. Il n'est plus question de réforme ni de création de nouveaux « départements », mais d'une évolution favorisant la convergence entre ceux qui existent. L'absence de recrutement d'enseignants, en particulier dans les universités publiques, favorise la mobilité de professeurs de plus en plus nombreux vers ces nouveaux programmes. D'ailleurs, l'establishment

${ }^{1}$ Les comités d'évaluation sont identifiés par le sigle CA qui, au CNPq, signifie "Comité assesseur » et, à la CAPES, «Commission de domaine ». CAPES : Coordination de perfectionnement du personnel de niveau supérieur. Rattachée au ministère de l'Éducation, elle intervient comme une agence d'accréditation et d'évaluation des cours hiérarchisés dans un classement. Elle est également responsable de l'octroi de bourses d'études (25000 en 2005) aux élèves de master et de doctorat. CNPq : Conseil national de développement scientifique et technologique. Rattaché au ministère de la Science et de la Technologie, sa mission est de promouvoir la recherche et la formation de chercheurs. Il accorde également des bourses aux élèves de master et de doctorat (12000 en 2005).

${ }^{2}$ D'autres expériences peuvent également être évoquées comme des éléments précurseurs au débat sur l'institutionnalisation de l'interdisciplinarité. C'est le cas de croisements typiquement «bidisciplinaires », tels que l'ingénierie biomédicale. 
universitaire ne s'y trompe pas et réagit très mal à ce jeu de vases communicants.

L'évaluation des enseignements de master et de doctorat menée par la CAPES permet d'en mesurer l'importance. Les programmes pluridisciplinaires augmentent de façon significative, représentant une part de plus en plus élevée des offres d'enseignement de $2^{\mathrm{e}}$ et de $3^{\mathrm{e}}$ cycle : de 1996 à 2004, ces masters et ces doctorats passent respectivement de 25 à 130 et de 7 à 32. Durant cette même période, le total de cours évalués, tous domaines confondus, a progressé de 1083 à 1927 masters et de 541 à 1034 doctorats. Alors que le taux de croissance annuel général des enseignements de master était de 8,3\%, celui des masters pluridisciplinaires était de 24,9\%. Dans le cas du doctorat, l'accroissement annuel était de 9,1\% pour l'ensemble et de $24,9 \%$ pour le groupe interdisciplinaire (CAPES, 2005).

Suivant les données recensées dans le document Plano Nacional de Pós-Graduação 2005-2010 ( Plan national des enseignements de $2^{\mathrm{e}}$ et de $3^{\mathrm{e}}$ cycle 2005-2010» [CAPES, 2005]), les estimations de croissance des enseignements de $2^{\mathrm{e}}$ et de $3^{\mathrm{e}}$ cycle au Brésil présentent le grand domaine «Multidisciplinaire et Enseignement » comme celui dont la croissance sera la plus forte, puisque sur $76 \%$ d'augmentation de l'offre totale d'enseignement, est attendue une augmentation de $122 \%$ pour les programmes interdisciplinaires.

\section{L'environnement dans le contexte universitaire}

La thématique «environnement », vue sous l'angle du développement durable, n'entre dans la formation universitaire que récemment, ce qui n'est pas propre au Brésil. Comme le monde des politiques publiques, le monde universitaire adopte rapidement, par une sorte de mouvement d'onde, une innovation, si elle s'impose dans un contexte donné. Ainsi, après un $X^{\mathrm{e}}$ siècle marqué par une spécialisation universitaire croissante, on assiste, à la fin du siècle, à l'apparition d'une dynamique nouvelle. Recherche et formation s'emparent de la thématique environnementale en réponse à des alarmes liées à l'explosion démographique, à la pollution industrielle, aux changements climatiques et à l'épuisement des ressources énergétiques... Parallèlement, de nouveaux mouvements sociaux ( «l'environnementalisme ») émergent, mettant en valeur des thèses pacifistes, des appels pour une meilleure qualité de vie, ou bien mobilisant autour de débats promus par des organismes multilatéraux, notamment la Conférence de Stockholm en 1972. Face aux nouveaux enjeux, les chercheurs sont interpellés sur leur capacité à proposer des solutions scientifiques et techniques.

On peut suivre sur une vingtaine d'années l'intégration progressive de ces nouvelles thématiques dans les institutions universitaires et de recherche brésiliennes. Grâce à la base de données SELAP (système en ligne de suivi de projets) du $\mathrm{CNPq}^{3}$, dont la dernière mise à jour date de 1985, on peut analyser l'activité de 54000 chercheurs et 36000 projets (Bursztyn, 1990).

En 1985, la base recense dans la catégorie « Environnement et ressources naturelles » 580 recherches, réparties selon les thèmes suivants : ressources naturelles (24\%); études intégrées des écosystèmes $(9 \%)$; état de l'environnement $(15 \%)$; aires protégées (3\%); polluants (2\%); dispersion et circulation des polluants $(6 \%)$; pollution $(21 \%)$; méthodes indirectes de contrôle de la pollution (11\%); droit environnemental et législation (4\%); éducation environnementale et formation de ressources humaines $(1 \%)$; systèmes d'information sur l'environnement et les ressources naturelles (3\%). Trois disciplines dominent, représentant 95,9\% du total : ingénieries (notamment sanitaire), biologie et chimie. Les domaines économique, social et politique, par exemple, n'apparaissent pas dans ces statistiques. Quant à la participation des recherches en "environnement et ressources naturelles ", elle s'élève, dans l'ensemble des recherches enregistrées au SELAP/CNPq en 1985, à seulement 1,6 \%, le pourcentage de chercheurs rattachés à ces recherches étant de $3,78 \%$.

En 2005, le panorama est bien différent. La banque de données de la CAPES (postérieure à 1987) ${ }^{4}$ comptabilise plus de 200000 thèses (avec résumé disponible), la plateforme Lattes de curricula vitarum $^{5}$, plus de 600000 chercheurs, le fichier du $\mathrm{CNPq}$, près de 15000 groupes de recherche. On remarque immédiatement que les relations avec des thématiques environnementales sont réparties de façon équitable entre les différents domaines de connaissances, les sciences humaines et sociales faisant progressivement leur apparition et les mots-clés qui leur sont associés étant présents dans quasiment tous les domaines du savoir (Tab.). La conférence Rio-92 («Eco-92») a entre-temps mobilisé et dynamisé l'intérêt de la société en général - et de l'Université en particulier - sur les grands problèmes environnementaux de la planète. On en voit la trace dans les médias avant la conférence, engouement qui retombera quelque peu par la suite.

Dans le cas de l'Université, même si la question environnementale a fortement marqué les débats depuis les années soixante - soit par des alertes néomalthusiennes (Hardin, 1968; Ehrlich, 1971), soit par des propositions

\footnotetext{
${ }^{3}$ C'est la plus ancienne base de données sur la production scientifique et technologique disponible au Brésil. Même s'il faut en citer les informations avec précaution, étant donné qu'elles ne prennent pas en compte la totalité des chercheurs du pays et que les moyens de collecte de données adoptés ne sont pas entièrement fiables, le système peut être considéré comme base de référence.

4 Cf. http://www.capes.gov.br

${ }^{5}$ Cf. http://lattes.cnpq.br (consulté le 20/11/2004).
} 
Tableau. Références aux principaux mots-clés dans le champ des questions environnementales dans les projets de recherche du CNPq (Source : www.cnpq.br).

\begin{tabular}{lcccc}
\hline Mots-clés & $\begin{array}{c}\text { Développement } \\
\text { durable }\end{array}$ & $\begin{array}{c}\text { Ressources naturelles } \\
\text { naturelles }\end{array}$ & Environnement & Biodiversité \\
\cline { 2 - 5 } Domaines & $\%$ & $\%$ & $\%$ & $\%$ \\
\hline Sciences agraires & 5,74 & 40,00 & 6,85 & 18,52 \\
Sciences biologiques & 11,48 & 7,06 & 10,27 & $\mathbf{6 6 , 0 5}$ \\
Sciences de la santé & 1,64 & 1,18 & 3,42 & 1,23 \\
Sciences exactes et sciences de la Terre & 4,92 & 20,00 & 20,54 & 9,88 \\
Sciences humaines & 23,77 & 11,76 & 19,8 & 1,23 \\
Sciences sociales appliquées & $\mathbf{3 6 , 0 7}$ & 17,65 & $\mathbf{2 2 , 7 4}$ & 2,47 \\
Ingénieries & 16,39 & 2,353 & 16,38 & 0,62 \\
\hline Total \% & 100 & 100 & 100 & 100 \\
\hline
\end{tabular}

de changement des paradigmes industriels -, un double mouvement a commencé à se produire durant la dernière décennie $d u X X^{e}$ siècle $: l^{\prime}$ « environnementalisation des disciplines » et $l^{\prime}$ « interdisciplinarisation de l'environnement ». Ce double mouvement sous-tend deux types d'arrangements institutionnels - l'un étant bien plus simple que l'autre - qui ont tendance à être complémentaires. Le premier entraîne un réaménagement au sein des départements disciplinaires qui, soit s'enrichissent de la thématique environnementale (l'adjectif étant accolé à la discipline), soit modifient leur structuration interne, comme c'est le cas pour la consécration de l'écologie dans la biologie ou de l'agroécologie dans l'agronomie. Le second se traduit dans l'apparition de programmes interdisciplinaires, notamment au niveau des cours de $2^{\mathrm{e}}$ et de $3^{\mathrm{e}}$ cycle. Ce deuxième mouvement retient notre attention, tout particulièrement à cause de son originalité, des enjeux qu'il soulève et de son rôle en tant que modèle institutionnel éventuel.

\section{Une typologie d'expériences}

Après des décennies de spécialisation, de fragmentation et de cloisonnement des disciplines, la thématique environnementale suscite un mouvement inverse d'ouverture. En soi, la nature et la complexité des problèmes à traiter dans l'univers des thématiques environnementales - synergies, objets en perpétuelle évolution, aspects englobés, intérêts impliqués, superposition d'échelles, révision de paradigmes consacrés, etc. - imposent que les compétences à mobiliser soient larges. L'originalité réside dans le fait que les nouveaux programmes interdisciplinaires font leur apparition à contre-courant de la vague de spécialisation qui a marqué la recherche scientifique en général, et l'Université en particulier, durant plusieurs décennies. Bien évidemment, un mouvement de ce type n'est possible qu'au moyen d'un apprentissage institutionnel ${ }^{6}$.

\footnotetext{
${ }^{6}$ Il coïncide avec la publication du Rapport Brundtland, en 1987 (World Commission of Environment and Development, 1987).
}

L'université de São Paulo est pionnière avec le PROCAM, Programme de sciences environnementales - vers 1990. En 1994, le programme Environnement et Développement débute à l'université fédérale du Paraná. Quant au programme Développement durable de l'université de Brasília, il est créé en 1995. En 2004, alors que plus de 30 programmes sont développés avec l'accréditation de la CAPES, il est possible d'établir une typologie du processus d'institutionnalisation de ces expériences, toutes sui generis.

\section{Formule « fait accompli »}

Le modèle USP (université de São Paulo) consiste en une interface entre enseignants et chercheurs à partir de la création d'un master. Le PROCAM fonctionne autour d'un groupe de professeurs, tous attachés à d'autres départements, avec une coordination alternée : tantôt sciences humaines, tantôt sciences naturelles ou biologiques. Université jouissant d'un grand prestige national, l'USP peut se permettre une telle innovation, car il est rare qu'elle soit disqualifiée ou déclassée sur le plan académique. C'est donc une logique de fait accompli qui prévaut, avec mise en place du programme pour en obtenir l'accréditation par la suite. Il lui faudra près de 10 ans pour avoir l'approbation de la CAPES. Durant cette période, de nombreux diplômes ont donc été délivrés sans l'homologation requise par le ministère de l'Éducation.

\section{Formule « silencieuse »}

Le modèle UFPR (université fédérale du Paraná) est singulier en ce qu'il s'est construit autour d'un projet de doctorat. Le double rattachement des enseignants (simultanément à des départements disciplinaires et au programme interdisciplinaire) a ouvert un espace interdisciplinaire reposant sur une organisation transversale complexe des formations, typique des grandes universités brésiliennes. Ce modèle, en situation de marginalité, s'est ainsi protégé des pressions et des oppositions 
émanant des départements institutionnalisés depuis plus longtemps. Autrement dit, l'existence du programme est liée, au moins partiellement, à la discrétion de son affichage dans le contexte institutionnel de l'université, même si, dans le court terme, son impact en a été diminué.

Le programme a bénéficié dans un premier temps d'un réseau international de coopération avec la France, qui a donné une légitimité à l'enseignement et apporté des ressources (humaines, essentiellement). Puis la classification du programme Environnement et Développement (MAD) de l'UFPR en tant que « chaire de développement durable » de l'Unesco l'a consolidé. Après quelques années, et malgré les difficultés de négociation d'une double affectation d'enseignants, le programme s'est enraciné (Zanoni et al., 2005).

\section{Formule « décollage sans retour »}

Le programme Développement durable de l'université de Brasília (CDS/UnB) est construit, comme dans le cas du Paraná, à partir d'un doctorat et s'appuie sur trois soutiens : celui de la présidence de l'université de Brasília, celui de la CAPES qui l'a reconnu préalablement, celui du CNPq qui a financé un programme spécial, le PADCT (Programme de soutien au développement scientifique et technologique).

Rapidement, des oppositions ont surgi de la part des départements d'origine des enseignants. Dans un contexte général de gel des embauches par concours dans la fonction publique, ceux-ci n'admettaient pas la perte partielle de leurs cadres sans qu'une substitution, à travers de nouveaux recrutements, ne soit mise en place. L'option adoptée fut celle de la «fuite en avant». Concrètement, cela s'est traduit par une augmentation du nombre d'élèves inscrits, une communauté plus impliquée et une portée plus large des actions réalisées. Pour qu'il soit viable, le programme choisit de montrer des résultats évaluables, très rapidement et en volume croissant, de façon à assurer sa pérennité.

Ayant commencé avec un doctorat modeste, il a rapidement engendré trois autres formations : en politique et gestion environnementale (un master recherche et un professionnel), en politique et gestion de la science et de la technologie (un master professionnel). L'ampleur du programme, qui a grandi de façon rapide et audacieuse, tout en acquérant de la visibilité, est l'un des facteurs expliquant sa légitimation.

\section{Formule «nous vaincrons ensemble»}

Depuis le début des années quatre-vingt-dix, un groupe d'universités de la région Nordeste se battait pour l'institution d'un programme en réseau. Voici un cas typique d'initiative avant la lettre, puisque le concept de réseau ne sera institutionnalisé que plus tard. En quête d'une alliance qui puisse les rendre suffisamment forts pour obtenir l'accréditation de la CAPES, plusieurs départements profitent des avantages comparatifs de chaque institution participante pour créer un programme de master interuniversités : le PRODEMA (Programme de développement et environnement), regroupant sept institutions ${ }^{7}$.

De ce groupe d'universités naît une certaine culture collective, mais avec des rattachements plus formels que réels. Bien que ces universités travaillent officiellement en réseau sous une même dénomination, elles conservent dans la pratique une autonomie certaine, due, en grande partie, au fait que l'évaluation et l'accréditation de la CAPES sont dispensées séparément.

\section{Formule « de transition »}

C'est le cas du Centre de hautes études de l'Amazonie (NAEA) de l'UFPA (université fédérale du Pará) qui, en près de vingt ans, est devenu un pôle de référence dans la région. Progressivement, le programme glisse du sous-domaine de la « sociologie», selon la classification de la CAPES, vers celui de la "pluridisciplinarité ». Sa structure institutionnelle s'est consolidée au fil du temps, au sein d'une université dans laquelle les pressions des départements étaient peu contraignantes. Aussi, la transition du NAEA vers une orientation socioenvironnementale ne pose pas de problèmes majeurs.

\section{Formule « conception naturelle »}

Depuis la fin des années quatre-vingt-dix, les universités, aussi bien publiques que privées, adoptent des programmes réunissant les thématiques environnementales et des démarches interdisciplinaires. Les universités plus jeunes, moins marquées par des tensions corporatistes internes, ont trouvé dans les programmes interdisciplinaires un véritable stimulant pour répondre à une forte demande de formation environnementale. Les cours qui en résultent collent aux problèmes plus spécifiques d'une région. Dans ce cas de figure, la naissance est naturelle, sans traumatismes, exception faite de la difficulté d'accréditation auprès de la CAPES. À titre d'exemple de ce type de formule, on peut citer les expériences de l'UFPE (université fédérale de Pernambuco) et de l'UFAM (université fédérale d'Amazonas).

\footnotetext{
${ }^{7}$ Universités impliquées dans PRODEMA : l'UFAL (université fédérale de Alagoas), l'UFSE (université fédérale de Sergipe), l'UERN (université de l'État de Rio Grande do Norte), l'UFPB (université fédérale de la Paraíba), l'UFC (université fédérale du Ceará), l'UESC (université de l'État de Santa Cruz) et l'UFRPE (Université fédérale rurale du Pernambuco).
} 


\section{Enjeux et problèmes liés à l'institutionnalisation}

Quel que soit son processus d'institutionnalisation, le mode opératoire des programmes interdisciplinaires de cours de $2^{\mathrm{e}}$ et de $3^{\mathrm{e}}$ cycle relatifs à l'environnement implique des difficultés et des obstacles, aussi bien sur le plan de la légitimation au sein de l'université de rattachement que du point de vue de l'accréditation et de l'évaluation de la CAPES. Il est possible de caractériser les obstacles affrontés par les tentatives d'organisation de communautés universitaires autour de la question environnementale comme un ensemble de syndromes (Bursztyn, 2005a), liés à des pathologies récurrentes présentant des symptômes très typés.

\section{Le syndrome de l'évaluation par des « impairs »}

En 2004, la CAPES a évalué un total de 2861 cours de master et de doctorat accrédités au Brésil. Sur ce total, les 111 cours classés dans le domaine «pluridisciplinarité et enseignement de sciences » ont la particularité d'avoir été analysés par des commissions d'évaluation pluridisciplinaires.

En 1999, la CAPES avait créé une commission spéciale pour évaluer les enseignements qui n'appartenaient à aucun des 8 domaines et des 44 sous-domaines de connaissance qui composent la structure du système d'évaluation. L'idée était de créer un espace pour le nombre croissant de programmes qui échappaient à la répartition traditionnelle par compartiments ou qui n'étaient pas assimilés ou accueillis par les commissions auxquelles ils avaient demandé leur rattachement ${ }^{8}$. Autrement dit, certains enseignements ne pouvaient relever d'une évaluation par les pairs (peer evaluation). Afin de constituer le groupe «pluridisciplinaire » et un ensemble d'enseignements qui, de par leur nature, étaient difficiles à classer dans les domaines disciplinaires existants, une nouvelle commission de domaine est alors créée : $l^{\prime}$ «enseignement de sciences ». Celle-ci ne traite pas d'une science particulière, ni uniquement de l'éducation.

Entre 1999 et 2003, l'évolution du nombre de programmes évalués par le domaine pluridisciplinaire a connu une progression élevée : 44 en 1999, 57 en 2000, 70 en 2001, 85 en 2002 et 111 en 2003. L'augmentation durant cette période a donc atteint $152 \%$, tandis que la croissance du nombre de programmes accrédités par la CAPES, tous domaines confondus, n'était que de $27 \%$. Il faut souligner que le sous-groupe de cours qui appartenaient à la thématique environnementale (classée avec

\footnotetext{
${ }^{8}$ Parmi les 23 membres de la commission qui a évalué les cours pluridisciplinaires en 2004, 8 seulement étaient des enseignants effectivement attachés à des programmes évalués dans ce domaine; les autres venaient de disciplines soumises à une évaluation dans d'autres comités de la CAPES.
}

les thématiques agraires) a connu une croissance proportionnellement supérieure à celle du groupe des cours pluridisciplinaires : passant de $25 \%$ du total, lors de l'évaluation de 2001, à $35 \%$ en 2002.

Certaines caractéristiques opérationnelles de la commission évaluant ces cours méritent ici d'être considérées. Tout d'abord, on constatera que la demande en accréditation de programmes est forte. Deux explications peuvent être avancées : la première relève de l'autoidentification de la part des institutions impliquées; la seconde résulte de la décision de commissions qui s'estiment incompétentes pour des cours relevant de la pluridisciplinarité. En conséquence, la commission d'évaluation est face à une surcharge de travail et n'accepte pas toujours les demandes de rattachement relevant pourtant de sa responsabilité. Ainsi, sur les 140 nouvelles demandes d'accréditation en 2003, 30 à 40 ont été renvoyées vers d'autres domaines, 60 à 70 ont été refusées et 40 seulement ont été approuvées. Un indice d'approbation inférieur à $30 \%$ reflète, sans aucun doute, une sélection que n'applique aucun autre domaine.

Par ailleurs, les évaluateurs du domaine pluridisciplinaire considèrent que leur fonction est celle $\mathrm{d}^{\prime} \mathrm{un}$ « incubateur de cours », qu'il faut orienter de façon adéquate, dès leur création, pour ensuite les rediriger vers un domaine consolidé. Vue sous cet angle, l'évaluation serait également un processus de formatage et d'orientation disciplinaire. C'est ce qui ressort du Documento da Área Multidisciplinar («Document du domaine multidisciplinaire ») de $2001^{9}:$ : [...] un nombre substantiel de cours sont transitoires au comité interdisciplinaire, ils devront être incorporés à d'autres comités » (sic).

Il convient ici d'évoquer Protágoras (philosophe grec du Ve siècle av. J.-C.), qui affirmait : « l'Homme est la mesure de toutes choses ». Ainsi, nous prenons connaissance $\mathrm{du}$ monde avec notre propre regard et nous finissons par évaluer les autres comme s'il s'agissait de nous. Dans l'évaluation de l'interdisciplinarité, la formation d'un «nous» fait encore défaut.

\section{Le syndrome de Narcisse ou des métriques externes}

Corollaire de l'évaluation par les «impairs », les critères définissant l'évaluation de la qualité des résultats sont fortement influencés par les idiosyncrasies propres aux domaines d'appartenance des évaluateurs. Domaine aux caractéristiques et aux identités propres encore mal comprises, l'interdisciplinarité finit par être sujette à un amalgame d'attentes de chacun des membres du comité. Ceci se reflète aussi bien dans le poids relatif attribué aux vecteurs de diffusion des résultats que dans l'analyse des maquettes des cours.

\footnotetext{
${ }^{9}$ Cf. http://www.capes.gov.br
} 
Tel Narcisse, qui (pour parodier le poète Caetano Veloso) «ne voit la beauté que dans le miroir», les évaluateurs ont tendance à valoriser davantage les interfaces des programmes analysés par rapport à leurs identités disciplinaires, refoulant les autres dimensions abordées. Dans le cas des règles qui instruisent le système d'attribution de note de la production académique, l'évaluation par les « impairs » amène une sous-valorisation de résultats qui pourraient être remarquables. Un exemple connu est la différence existant entre les sciences humaines et les sciences naturelles et exactes quant au type de publications visées pour la diffusion de résultats. Le premier groupe valorise davantage les livres, alors que le second privilégie les articles publiés dans des revues spécialisées. Bien entendu, ceci n'implique nullement que l'un soit meilleur ou pire que l'autre; ils sont juste d'une nature différente. Quand chacun de ces domaines est évalué par ses propres pairs, la différenciation n'amène pas de malaise. Mais lorsque les cours interdisciplinaires sont évalués par des critères et des pratiques propres à des disciplines spécifiques, le résultat est fatalement injuste, avec des reproches souvent inappropriés et très peu de considération pour les résultats obtenus au sein de la discipline.

\section{Le syndrome ARI (aucune des réponses indiquées)}

En conséquence, on constate une certaine tendance à rediriger vers les programmes interdisciplinaires tous les cours et programmes de recherche qui ne cadrent pas directement avec l'un des domaines officiellement reconnus par la CAPES. Par cette rigidité de classement, les cours pluridisciplinaires paraissent échapper à toute classification et, avec eux, les objets traités. C'est comme s'il s'agissait de l'option "aucune des réponses indiquées ». Si ce syndrome persiste et se renforce, il y a un grand risque de crise d'identité des programmes en question.

\section{Pour conclure : en quête d'un modèle institutionnel}

L'Université brésilienne est en crise. Telle est la position consensuelle qui rallie le plus grand nombre d'avis des différents protagonistes de sa mission. Elle est en crise, car elle ne parvient plus à être comme elle était, sans réussir à devenir autre chose. C'est une crise existentielle qui se répercute également sur son avenir. Il est donc temps de partir en quête d'alternatives.

$C^{\prime}$ est dans cette optique que les processus d'institutionnalisation que connaissent actuellement les cours relevant du domaine pluridisciplinaire doivent faire l'objet d'une évaluation précise : de la ligne directrice jusqu'aux modèles d'implantation. Nés dans une adversité liée à un défaut de légitimité institutionnelle et de moyens, ce sont des expériences remarquables d'un métabolisme novateur. Non seulement parce qu'ils innovent sur le mode opératoire pour faire face aux questions complexes et provocatrices de l'actualité, mais aussi parce qu'ils sont des structures créatives au sein d'une organisation administrative figée.

Les partenariats issus des expériences interdisciplinaires doivent aussi être évalués comme de nouvelles pistes à explorer. Certains faits peuvent nous aider à maintenir ce cap, si l'on veut progresser dans la réflexion sur le dessein, possible et souhaitable, à assumer par une Université d'après-crise :

- le domaine «multidisciplinaire » de la CAPES est celui qui croît le plus rapidement, même si on considère que, dans son ensemble, le système brésilien des formations de $2^{\mathrm{e}}$ et de $3^{\mathrm{e}}$ cycle progresse à un rythme élevé;

- les demandes de formation à travers des programmes de $2^{\mathrm{e}}$ et de $3^{\mathrm{e}}$ cycle basés sur l'environnement ne cessent d'augmenter;

- certains programmes se sont déjà consolidés après 10 ans ;

- une culture du "champ», selon la proposition de Bourdieu (1983 et 1990) ${ }^{10}$, est en train de naître. Contrairement à ce qui se passe dans d'autres domaines, il existe un large espace pour la solidarité, avec moins de compétition. La collaboration est une caractéristique des rapports existant entre les différents acteurs qui interviennent dans le domaine multidisciplinaire;

- une autosuffisance relative existe, puisqu'il s'agit de programmes nés en pleine crise;

- l'institution de la double affectation favorise un caractère organique, au sein d'une Université fragmentée. Le "modèle en étoile " (un noyau avec divers satellites) semble efficace, puisqu'il permet à chaque enseignant ou chaque chercheur de militer auprès de son département. Dans le même temps, il crée un espace de cohabitation interdisciplinaire. Contrairement aux arguments avancés pour justifier l'opposition des départements d'origine, cette pratique apporte beaucoup à ces derniers dans la mesure où elle leur propose de nouveaux intrants qui interpellent et enrichissent leurs routines (Bursztyn, 2005b) ;

- il y a une forte propension à associer la théorie à la pratique, ainsi qu'à récupérer dans la pratique universitaire la fonction de vulgarisation que l'Université a délaissée (Buarque, 1994).

\footnotetext{
10 «Un terrain se définit par les objets de disputes et les intérêts spécifiques irréductibles aux objets de disputes et aux intérêts propres d'autres terrains et qui ne sont pas perçus par celui qui n'a pas été formé pour pénétrer ce terrain » (Bourdieu, 1983).
} 
Enfin, à l'image des propositions de Castells (1999) pour la structuration de l'État dans une ère postnéolibérale - l'État-réseau -, l'Université d'après-crise pourrait devenir une Université-réseau. Au lieu d'une compartimentation en départements imperméables, qui ne communiquent pas entre eux et perdent leur capacité à faire face à certains enjeux de la modernité - ceux-ci requérant des solutions complexes et interdisciplinaires -, un fonctionnement en réseau pourrait rendre viable un mouvement axé sur l'intégration.

$C^{\prime}$ est dans ce sens que, pour en revenir aux deux mouvements évoqués au début («environnementalisation des disciplines » et «interdisciplinarisation de l'environnement »), il ne faut pas succomber aux pressions visant à «disciplinariser l'environnement ».

\section{Références}

Bourdieu, P., 1983. Algumas propriedades do campo, in Bourdieu, P. (Ed.), Questões de Sociologia, Rio de Janeiro, Marco Zero, 89-94.

Bourdieu, P., 1990. O campo intelectual: um mundo a parte, in Bourdieu, P. (Ed.), Coisas Ditas, São Paulo, Brasiliense, 68-180.
Buarque, C., 1994. A Aventura da Universidade, São Paulo, Paz e Terra/Unesp.

Bursztyn, M., 1990. Ciência, Tecnologia e Pesquisa Ambiental na Universidade Brasileira : dilemas e potencialidades, Seminário Universidade de Meio Ambiente: Documentos Básicos, Brasília, IBAMA, 243-253.

Bursztyn, M., 2005a. Meio ambiente e Interdisciplinaridade: desafios ao mundo acadêmico, Revista Desenvolvimento e Meio Ambiente, 10,2, 67-76.

Bursztyn, M., 2005b. A institucionalização da interdisciplinaridade e a universidade brasileira, Revista Liinc, 1, 2, 38-52.

CAPES, 2005. Plano Nacional de Pós-Graduação (PNPG) 20052010, Brasília, Capes/MEC ; cf. www.capes.gov.br (consulté le $10 / 1 / 2005$ ).

Castells, M., 1999. A Sociedade em Rede, São Paulo, Paz e Terra, vol. I.

Ehrlich, P., 1971. The Population Bomb, New York, Ballantine Books.

Hardin, G., 1968. The Tragedy of the Commons, Science, 162, $3859,1243-1248$

Morin, E., 2004. La Méthode, 6. Éthique, Paris, Le Seuil.

World Commission on Environment and Development, 1987. Our Common Future, Oxford, Oxford University Press.

Zanoni, M., Raynaut, C., Mendonça, F., 2005. Une expérience de formation interdisciplinaire aux recherches sur le développement durable : la chaire Unesco de l'Université fédérale du Paraná (Curitiba, Brésil), Natures Sciences Sociétés, 13, 2, 198-205. 